\title{
A Scenario-Based Approach To Predict Energy Demand And Carbon Emission of Electric Vehicles On The Electric Grid
}

Wai Cheung ( $\nabla$ wai.m.cheung@northumbria.ac.uk)

University of Northumbria https://orcid.org/0000-0003-0051-2615

\section{Research Article}

Keywords: Electric vehicles, Electric grid, Energy demand, C02 emissions, Green vehicles, UK electric grid infrastructure Posted Date: November 22nd, 2021

DOI: https://doi.org/10.21203/rs.3.rs-974754/v1

License: @ (i) This work is licensed under a Creative Commons Attribution 4.0 International License. Read Full License 


\section{Abstract}

UK plans to ban the sale of new diesel and petrol cars by 2030 to be replaced by electric vehicles (EVs). However, motoring experts warn that this demand for electricity will increase by $50 \%$ which will place unprecedented strain on the UK's National Grid. The question is, will the UK's electric grid infrastructure ready for this change? This comparative study investigates into the effect of UK green vehicles have on the electricity grid and will present a new insight into improving their environmental impact to the electric grid. This work is carried out with relevant data from 2014 to 2030 and addresses the carbon dioxide emissions produced on the natural environment and how EVs can help to reduce such pollution. This investigation will assess the effects on the electricity grid with or without EVs from an environmental, economic and social viewpoint. Recommendations from this work will help the industry to make key decisions of how to cope with demand and requirements to make a smart grid environment work.

\section{Introduction}

In 2018, an estimated over 1 billion cars were in existence around the world; their widespread use has had extensive environmental and economic impacts (Takács et al., 2018). To power all of these vehicles $94 \%$ of the energy required $\mathrm{CO}_{2}$ intensive source such as oil (Köne and Büke., 2010). In 2007 road transport was responsible for approximately 17\% of global $\mathrm{CO}_{2}$ emissions. Passenger vehicles are expected to consume around $44 \%$ of all energy consumed in transport through 2050 (Paladugula et al., 2018).

Reducing the carbon dioxide emissions in the transportation sector is of global importance. The UK National Grid aims to reduce all carbon emissions by $80 \%$ by 2050 (Liu et al., 2020). With increasing amounts of cars on the roads, improving their efficiency is crucial, and electric vehicles (EVs) are widely discussed as one of the main future technologies to combat environmental impacts. EVs have a battery storage system of $28.9 \mathrm{kWh}$ or more, a means of recharging the battery from an external source, and the ability to drive at a range of 350 miles (Park., 2018). There are a range of passenger EVs being developed by manufacturers. These vehicles range from medium sized vehicles such as the Nissan Leaf to Sport and luxury vehicles such as the Tesla model $S$; these vehicles require different amounts of energy from the electric grid to be charged.

It is predicted that the market share of EVs in the future will be extensive with $25 \%$ of all newly purchased vehicles being EVs a from 5 million EVs in 2018 increasing to over 40 million in 2030 globally (Global EV Outlook., 2019). Vast research has been carried out into EVs but there has been minimal research into the impacts of these vehicles will have on the electricity grid (Kapustin and Grushevenko., 2020). Significant impacts are expected to the power distribution grid due to the high energy capacity these vehicles will require. Resultant effects on the electricity grid will dictate the design of EVs interfaces and the way in which power networks will be designed and controlled.

Green vehicle implementation introduces complexities into the grid. One reason being there is no way of predicting when and where the vehicles will require energy from the electric grid. Additional battery load may occur at times when the electricity supply system is already heavily loaded. In this scenario the impacts will be across the entire power system; therefore, the impacts of EVs need to be evaluated long before they become heavily embedded into the transportation sector.

This research evaluates the pressures that EVs will place on the electric grid. The study assesses the loads that will be applied to the electric grid as a result of market development of the vehicles with respect to a solution for lessening environmental impacts. Results gained from this work highlights future considerations of requirements of a smart grid system.

\section{Literature Review}


A plug-in hybrid is an electric vehicle (PHEV) that can be plugged into the electricity grid and can be driven by electricity for at least 10 miles without consuming any gasoline (Martinez et al., 2016)]. These vehicles are able to run on fossil fuels, electricity or both leading to a range of advantages. Pressures of both environmental protection and energy conservation means the development of EVs have been accelerated worldwide (Adna et al., 2018).

$\mathrm{CO}_{2}$ emissions are the most commonly measured output to evaluate the environmental impacts of switching to EVs. From an environmental perspective, the replacement of internal combustion engine cars with EVs may be beneficial for the climate because of the possible reduction of greenhouse gas emissions (Thiel et al., 2010). Juul and Meibom (2011) calculate that the integration of electric power and transportation sectors in Denmark reduces transportation related $\mathrm{CO}_{2}$ emissions by $85 \%$. Vehicles using electricity from the energy mix produce $56 \%$ of savings in the production of $\mathrm{CO}_{2}$ compared to the modern spark ignition engines (Hromadko and Miler., 2012). Richardson (2013) finds that EVs reduce the total number of $\mathrm{CO}_{2}$ emissions, even in electricity systems with a high fraction of fossil fuel generation. This is due to the high efficiency of an electric motor compared to an internal combustion engine.

One criticism of EVs is that they simply transfer $\mathrm{CO}_{2}$ emissions from the vehicles exhaust to power plants (Razeghi et al., 2011). Air emissions resulting from electricity production depend on the fuel mix; this differs by country and varies over time ( Doucette and McCulloch., 2011). These differences in the type, size and location of emissions need to be weighted in order to give an overall picture of environmental impacts and external costs. There is an EU requirement for $20 \%$ of final energy to be derived from renewable resources by 2020; the target for the UK is 15\% (European Commission., 2020). A report in 2014 by National statistics said that the UKs gained $19.7 \%$ of its total energy from renewable resources (Renewables., 2015). This level is a huge improvement in comparison to previous years; however many other countries gain a much lower percentage of their energy from renewable resources. For example the Czech Republic only captures $2.15 \%$ of its energy from renewable sources (Hromadko and Miler., 2012). There is future research needed to reduce air pollutant emissions from electricity production. Electricity consumption does not emit $\mathrm{CO}_{2}$ at the point of use, greenhouse gas intensity $\left(\mathrm{gCO}_{2}-\mathrm{eq} / \mathrm{KWh}\right)$ of electricity used to charge vehicles is a key parameter to estimate life cycle greenhouse gas impact (Constantine et al., 2008).

The effects that EVs may have on the electricity grid are covered by Kapustin, N.O. and Grushevenko (2020). The article suggests that $\mathrm{EVs}$ are one of the best methods available to reduce current $\mathrm{CO}_{2}$ emission levels however there are some limiting factors. The impacts of EVs are determined by regional grid analysis. Centred by the number of EVs in a region, the demand for EVs, the other electrical needs in an area and how this affects the supply and demand for EVs in that region.

Kivilnova and Meibom (2011) analyse the significance of smart charging EVs compared to immediately charging when connected to the grid. McCarthy et al., (2008) developed a simplified dispatch model to investigate impacts of integrating EVs into California's energy system. The authors indicate that further research needs to be carried out into the affects a large number of EVs could have on a grid.

Shao et al (2009) investigated the impacts of charging EVs on a typical distribution network in Blacksburg, US. The investigation compares the results when the cars are charged at peak and off-peak times of a typical day. When the cars are charged in this mode at a peak time the transformer is overloaded and the efficiency of the transformer is reduced. The research suggests two strategies that could be implemented to prevent this overload; stagger charge and load control.

Perujo and Ciuffo (2009) evaluated the potential impacts to the grid for the province of Milan. The key features they suggest to investigate are the potential market penetration and the main technical features of the 'fleet'. Capacity of the vehicle, the range of travel, energy consumption and re-charging time are important factors to consider.

A report by Shafiee et al., (2013) investigated the impacts of changing levels of EVs on the electric grid. The authors found that if $10 \%$ of the current fleet of cars in the United States turned electric then the electrical load would increase by $31.35 \mathrm{GW}$. They concluded that the impacts of EVs on the grid are favourable, the research stresses the need to evaluate the effects of 
an increased green vehicle share. JP Morgan (2018) projected that by the year 2025 there could be 8.4 million EVs sold worldwide. The research carried out by $\mathrm{Li}$, and Wang., (2012) promotes the interest of China to establish itself as the largest producer of EVs, the government is funding over $\$ 1.5$ billion to help the industry. The research indicates that the number of EVs will increase due to many incentives.

Hadley and Tsvetkov (2009) create a scenario where the market share of EVs increases from $0 \%$ in 2010 to $25 \%$ in 2020. The model then plateaus at $25 \%$ for the next decade. The report shows that the increased penetration of vehicles puts added strains on the system. There is an increase in electricity demand, generation and price.

Market penetration for EVs is currently a topic of extensive research. To gain an idea the impact of increasing numbers of EVs in the UK this study considers three scenarios.

1. Slow-Progression of growth of EVs from 2014-2030. This scenario creates a situation where in 2030 EVs make up $10 \%$ of the car fleet in the UK.

2. Intermediate-Progression of growth of EVs from 2014-2030. This scenario creates a situation where in 2030 EVs make up $15 \%$ of the car fleet in the UK.

3. Fast-progression of growth of EVs from 2014-2030. This scenario creates a situation where in 2030 EVs make up $25 \%$ of the car fleet in the UK.

\section{Methodology}

To access the overall environmental impact that EVs have on the electricity grid certain factors are taken into account.

(i) To investigate the energy demands that an increased number of electric vehicles will apply to the current electricity system.

(ii) To evaluate the environmental impacts the research focuses on the carbon emissions produced by these vehicles, currently the UK has plans in place to reduce the $\mathrm{CO}_{2}$ emissions by $45 \%$ by 2020 (Sithole et al., 2016). Petrol and diesel cars will gradually be phased out by 2030 .

The method used in this work is accessed the success of large-scale deployment of EVs into the UK.

\subsection{Assessment of the impact of EVs on energy demand}

A scenario-based simulation tool is used to evaluate the impact of EVs in the future depending on the market penetration of the vehicles. Once the percentage of EVs has been predicted in the future in terms of the entire car fleet it is important to investigate detailed information about the car fleet itself. There are a number of factors that need to be explored including:

i) Total predicted number of cars in the 'car fleet'.

ii) Energy required recharging the 'car fleet'.

iii) Composition of the 'car fleet'.

To gain an idea of the demand that EVs will have on the electricity grid this research investigates at the yearly requirement of electricity that EVs will require at different market penetration levels. This scenario is based on the fact that all EVs will only be charged when they needed. The total number of charges daily can be calculated by dividing the range of the vehicle by the total miles travelled daily; this work assumes that EVs daily mileage for commuting trips is in the 20 miles range.

By looking at the daily energy demand in more detail it is possible to assess the impact that EVs will have on the electricity grid. Information of the daily energy demand will be compared to the demand placed on the grid by changing levels of 
market penetration.

a) The number of charges daily is multiplied by the number of vehicles for the total number of daily charges needed for the entire fleet.

b) By multiplying the value in (a) by the battery capacity and dividing by the battery efficiency of $90 \%$ it is possible to gain a value of the total electrical energy required.

c) A yearly total of electricity required by the fleet can be calculated by multiplying the daily energy required by 365 days.

Figure 1 shows the predicted energy consumption in the UK in 2030. This will act as a comparison to evaluate the impact on electricity demand due to EVs.

\subsection{Assessment of the impact of EVs on the energy demand}

When assessing the potential impact of EVs on the electrical grid and in particular the entire electric consumption a number of key factors have to be considered.

- The core technical features for the available cars need to be evaluated; from a short to medium term.

- The market penetration of the fleet of vehicles available needs to be estimated in terms of future evolution.

A study by Harris (2009) investigating the impact of the energy requirements of an increased number of EVs on the UK electricity grid in short and medium term. It is found that the grid capacity should be adequate for a $10 \%$ market penetration of EVs. However, as EVs are still in early stages of production it is hard to estimate future trends regarding the vehicles, market response will affect the vehicles development as well as technological advances. Harris (2009) also stated that local network problems could be an issue depending on distribution network capacity and concentration of EVs.

The technical features of the EVs in the future will determine potential market penetration. Factors that need to be considered are:

- the battery capacity of EVs and;

- the range or distance they can travel.

- their energy consumption per unit of distance covered.

All of these elements influence the type of commuter that will drive these vehicles. Currently the range of EVs regards as being particularly small; some vehicles barely reaching 100 miles (Wu et al., 2015). This means that the vehicles therefore need to be recharged more frequently and this process currently requires several hours depending on the energy available. Putrus et al., (2009) claim that slow charging from a single phase takes around 6 hours. Majority of the people that do not need to travel long distances are suited to these vehicles; satisfying their need to help the environment is also a factor to purchasing EVs. Due to the cars small range of travel this makes them very suitable for urban use. However large urban cities are highly energy consuming areas, this means they may substantially suffer from the electrical energy demand of these vehicles. The UK has been chosen as a case study because of its urbanised areas and potential market penetration in the future for EVs.

Table 1. Available EVs 


\begin{tabular}{llllll} 
Manufactures & $\begin{array}{l}\text { Type of } \\
\text { EVs }\end{array}$ & $\begin{array}{l}\text { Battery Capacity } \\
\text { (KWh) }\end{array}$ & $\begin{array}{l}\text { Time to Charge } \\
\text { (Hours) }\end{array}$ & $\begin{array}{l}\text { Distance Travel } \\
\text { (Miles) }\end{array}$ & $\begin{array}{l}\text { Consumption (KWh/100 } \\
\text { Miles) }\end{array}$ \\
\hline Nissan & LEAF & 24 & 5 & 124 & 19.35 \\
\hline Mitsubishi & Outlander & 12 & 5 & 34 & 35.29 \\
\hline BMW & i3 & 22 & 5 & 80 & 27.5 \\
\hline Renault & Zoe & 22 & 5 & 149 & 14.77 \\
\hline Tesla & Model S & 85 & 5 & 265 & 32.08 \\
\hline Kia & Soul & 27 & 5 & 132 & 20.45 \\
\hline Volkswagen & E-UP & 18.7 & 5 & 93 & 20.11 \\
\hline Ford & Focus- & 23 & 5 & 76 & 30.26 \\
\hline Audi & Electric & & 5 & 31 & 29.03
\end{tabular}

Table 1 shows a collection of EVs available in the UK during this research investigation. Table 2 shows a summarised classification of vehicles, the vehicles have been clustered into specialised groups depending on the capacity of the battery. The vehicle categories chosen for this study are small, medium and large. Table 2 includes the expected recharging times for the vehicles. Recharging time is very important as it helps to estimate the energy required by all of the EVs. The recharging times vary for each vehicle; for this study the investigation have considered an average recharging time of six hours. This time is expected to decrease due to technological developments.

Table 2. Simplified groups of EVs

\begin{tabular}{|c|c|c|c|c|c|}
\hline \multirow[t]{2}{*}{ Segment } & \multirow[t]{2}{*}{$\begin{array}{l}\text { Battery Capacity } \\
\text { (KWh) }\end{array}$} & \multicolumn{2}{|c|}{$\begin{array}{l}\text { Time to Charge } \\
\text { (Hours) }\end{array}$} & \multirow[t]{2}{*}{$\begin{array}{l}\text { Distance Travel } \\
\text { (Miles) }\end{array}$} & \multirow[t]{2}{*}{$\begin{array}{l}\text { Consumption (KWh/100 } \\
\text { Miles) }\end{array}$} \\
\hline & & Domestic & Fast & & \\
\hline Small & 15 & 6 & 1 & 50 & 30 \\
\hline Medium & 25 & 6 & 1 & 80 & 31.25 \\
\hline Large & 35 & 6 & 1 & 110 & 31.81 \\
\hline
\end{tabular}

The recharging power for each individual vehicle of a car fleet has been calculated as shown in Table 3. The power required to the grid is dependent on the type of charging available. Domestic charging requires a longer time to recharge but less electrical energy is needed from the grid to fully charge a vehicle. The fast charging option requires a considerably larger amount of energy from the electricity grid because it charges in a substantially shorter time.

Table 3. Estimated power required to recharge 1 vehicle

\begin{tabular}{lll} 
EVs & \multicolumn{2}{l}{ Electricity Required by Grid (KW) } \\
\hline & Domestic Charging & Fast Charging \\
\hline Small & 2.78 & 33.33 \\
\hline Medium & 4.63 & 55.56 \\
\hline Large & 6.48 & 77.78 \\
\hline
\end{tabular}

Furthermore, the li-lon battery efficiency needs to be taken into account of in order to understand how much electrical energy is required to the distribution grid. For this study a battery efficiency of $90 \%$ has been considered (Richardson et al., 2012). The battery efficiency discharge phase needs to be considered when looking at the range of EVs. 
The recharging power to recharge each vehicle is determined by equation (1):

Power required by grid to recharge $=$ Capacity $\times$ Time $\times 0.9$

A PHEV is a hybrid vehicle with rechargeable batteries by connecting to the grid and has an internal combustion engine that can be activated when the batteries need recharging [40]. PHEVs offer the range of existing hybrid vehicles but also offer potential cost savings to ensure the energy benefits of fully electric vehicles.

It is critical to note that this study does not include the impacts of PHEVs on the electricity grid. The study considers all of the typologies of electric vehicles as one commodity when looking at the future of the electric car fleet. This investigation includes PHEVs and fully battery operated vehicles as one due to the type of trips that are considered. Since EVs have a limited range, the cars in this study are considered to be driven in short distances mainly for working purposes. As the vehicles would not be travelling excessive distances the internal combustion engine of the hybrid vehicles would not be required when making daily trips. Therefore the vehicles will operate in a sense as fully electric vehicles and will require the same energy. Consequently the same category will be considered when looking at market penetration of these EVs. .

A huge uncertainty of this study is the potential market penetration of EVs in the UK. It is important to understand the amount of EVs that will be in the UK in the future to gain an indication of their impact on the electrical grid. From the literature reviews, it has become clear that this research has already undertaken on the growth of PHEVs. The development of fully battery operated vehicles is predicted to be slower than PHEVs; reasons for this inclusion that there currently are more PHEVs on the market than EVs. However these factors become irrelevant in this study as this investigation assumes that they are as one entity. Many degrees of freedom of the uncertainty of the vehicles evolution have a resultant impact of projecting the progression of EVs in the future. This study examines the market penetration of EVs from 2014-2030. The model created develops a scenario where in 2030 there will be a different percentage of electric cars depending on the success of the vehicles penetration into the market.

As this work investigates the impact on the electricity grid from the growth of EVs in the future, Transport Statistics Great Britain (2020) contains records of the number of registered cars in the UK dating from 1970-2015. From this information it is possible to predict a trend for the amount of cars in the future as illustrated in Figure 2. Figure 2 shows the real number of cars and the estimated number up to 2030. Incorporating the predicted number of cars in the future and using the three market penetration scenarios already stated it is possible to gain an estimate of the market share of EVs in the UK. It is important to note that the amount of cars predicted in the future is only an estimate.

This study focuses on the impact of EVs on the distribution grid it is important to consider the technical information about the future 'car fleet'. Electric cars have different battery capacities means different energy values are required by the electricity grid. Table 2 shows the three categories of electric fleet; small, medium and large. The composition of the vehicle fleet needs to be considered when investigating the total energy requirement of the EV fleet to the electric grid of the UK. By taking consideration of the new registrations by vehicle segment, it is possible to use the configuration specified in Table 4 to predict the amount of energy required by a future car fleet. To find out the total number of cars from each category the total number of cars predicted in that year is multiplied by the percentage stated in Table 4.

Table 4. Composition of EVs

\begin{tabular}{ll} 
Vehicles by Segment & Percentage (\%) of Electric Car Fleet \\
\hline Small & 35 \\
\hline Medium & 44 \\
\hline Large & 21 \\
\hline
\end{tabular}

Page $7 / 19$ 
To find out the impact that the vehicles will have on the electric supply system it is important to have comprehensive information about the system. Table 4 states the predicted energy required to fully charge a vehicle. The energy required to recharge each vehicle type can be determined by equation 2 . The calculation used in the study assumes that each vehicle in the system in fully charged once a day.

Energy $=$ Battery capacity / (Battery recharging time $\mathrm{x}$ Battery efficiency)

\subsection{Assessment of the impact electric vehicles will have of carbon emissions}

To assess the impact of EVs on carbon emissions by using data on the energy mix in the UK. As explained in the literature review EVs claim to have zero emissions but carbon is still emitted into the atmosphere as a result of electricity production. Therefore, to evaluate the impact of large deployment of EVs, the way in which electricity is produced will need to be investigated. Consequently, this research work will look at datasets that predict the way the UKs electricity production in the future. This part of the research work focuses on evaluating the impact EVs on the electricity grid in terms of reducing carbon emissions by considering different market penetrations of EVs. The factors that need to be taken account of in the prediction are:

1. Different market penetration levels of EVs will be considered ranging from $5 \%$ to $30 \%$.

2. Different carbon emission factors for the 'normal' vehicle fleet will be considered.

3. To calculate the carbon emissions produced by electricity generation a carbon emission factor needs to be used. This factor remains constant throughout the model. It converts $\mathrm{KWh}$ of electricity to $\mathrm{KgCO}_{2}$ per mile travelled per car.

4. The prediction uses a constant driving range of 20 miles.

\section{- Energy Mix in the UK}

In order to find out the impact of EVs on carbon emissions information regarding the UKs energy mix is necessary. The energy used to power the cars has been generated from a mix of production technologies which will produce a corresponding quantity of emissions. The energy mix contains information about what fuels are used to produce electricity and the percentage of usage.

\section{- Electricity_carbon Factor}

This work uses an electricity carbon factor to predict the carbon emissions produced from generating the electricity used to power the EVs. The electricity carbon factor converts the electricity needed to power the car fleet in KWh to carbon emissions produced $\left(\mathrm{KgCO}_{2}\right)$. For this work a carbon emission of 0.4585 was used which corresponds with the 2015 electricity carbon emission factor (International Electricity Factors., 2018). It is important to note that this carbon emission factor is an average and changes from year to year. Therefore this research predicts results that show an improved reduction of $\mathrm{CO}_{2}$ emissions as a result of an increased number of EVs in the future.

\section{- $\underline{\text { Range of miles }}$}

To access the impact of carbon emissions this project uses a driving range of 20 miles, this is because the study assumes that EVs will be used for commuting purposes. This driving range used is equivalent to the driving range used to access the impact of EVs on the electricity grid.

- -g $_{\underline{\mathrm{CO}}}-\underline{\text { per Km}}$

The carbon emissions per kilometre of travel are converted to find the overall $\mathrm{CO}_{2}$ impact. This study evaluates from the aspect that the $\mathrm{gCO}_{2}$ per kilometre per car will only improve. 


\section{Results}

\subsection{Results of electricity demand by EVs}

This work has examined the electricity demand of an increasing number of EVs from 2014 to 2030. Results of market penetration of EVs over time and the electricity the vehicles required from the predictive model are shown in Figure 3 . The evaluation predicts that in 2030 there will be different market penetration levels of EVs in the UK. In 2014 the percentage of EVs is $1 \%$ of the total cars in the UK, at this level the cars require between $1 \mathrm{GW}$ and $1.5 \mathrm{GW}$ of electricity to fully charge all of the vehicles. As indicated in the results it is possible to gain a visual appearance of the impact the cars will have on the electric grid.

The first scenario where there is a slow progression of EVs predicts an increase of $9 \%$ as a proportion of total cars from $1 \%$ of total cars in 2014 to $10 \%$ in 2030 . The results show there is a positive correlation between the percentage of EVs in the UK and the amount of electricity needed. The peak electricity required to charge the vehicles is in the year 2030; due to having a larger percentage of vehicles being electric. When there is a 10\% market penetration of cars in 2030 the total electricity to power them is approximately $16 \mathrm{GW}$. This value is over 12 times the electricity needed to charge the cars in 2014.

The second scenario accounts for an intermediate progression of cars in the UK from 2014 to 2030; increasing from 1\% in 2014 to $15 \%$ in 2030 . The amount of electricity needed to power the cars peaks in 2030 at approximately 24 GW.

The third scenario predicts a fast progression of electric cars in the UK; starting at $1 \%$ in 2014 and increasing to $25 \%$ in 2030. The fast progression scenario requires the largest amount of energy to recharge the cars. As indicated in the illustration, potentially, $40 \mathrm{GW}$ of electricity is required daily to charge EVs. This value is over thirty times larger than the electricity required for charging EVs in 2014. By comparing the energy required by the vehicles in Figure 4, it can be seen that the daily demand the vehicles require compared to the electricity demand currently.

Table 5 represents the results of the incidence of electric power demand on the yearly total consumption EV fleets share ranges from $1-30 \%$. When the EV fleet share is the lowest value of $1 \%$ the incidence on total electric energy consumption is the lowest; only requiring $0.26 \%$ of the yearly electric energy consumption. Even in the unlikely event when the EVs hold the largest share of $30 \%$ of total cars percentage incidence of total power only reaches $7.86 \%$.

Table 5

Incidence on total electrical energy consumption

\begin{tabular}{|c|c|c|c|c|c|c|c|}
\hline \multirow{2}{*}{$\begin{array}{l}\text { Year } 2030 \\
\text { Fleet Share (\%) }\end{array}$} & \multicolumn{7}{|c|}{$\begin{array}{l}\text { Predicted Total Electric Energy Consumption in the UK } 2030 \\
\text { (350 TWh) }\end{array}$} \\
\hline & 1 & 5 & 10 & 15 & 20 & 25 & 30 \\
\hline EV Consumption (x 10 ${ }^{8}$ ) & 9.18 & 45.9 & 91.8 & 138 & 184 & 230 & 275 \\
\hline $\begin{array}{l}\text { Incidence of EVs on Total Electric Energy } \\
\text { Consumption (\%) }\end{array}$ & 0.26 & 1.31 & 2.62 & 3.93 & 5.24 & 6.55 & 7.86 \\
\hline
\end{tabular}

\subsection{Results for calculating $\mathrm{CO} 2$ impact}

This work uses three scenarios for predicting the impact of EVs on the overall carbon emissions. There are two constant variables in the predicative model and these are: electricity carbon factor of $0.4585 \mathrm{gCO}_{2}$ and a distance of 20 miles. The independent variable used to evaluate the carbon emission impact from EVs is the carbon emissions produced by the 'normal' car fleet $\left(\mathrm{KgCO}_{2}\right.$ per $\left.\mathrm{Km}\right)$. 
(i) Figure 5 shows the result of the first scenario. The first scenario uses average car carbon emission data from the year 2000 where the total carbon dioxide produced is $181 \mathrm{gCO}_{2}$ per car per $\mathrm{Km}$. The total carbon emissions produced by the 'normal' fleet and the EVs are combined to gain a value for the total $\mathrm{CO}_{2}$ emissions produced. When there are no EVs present the total carbon emissions is greater with a value of $1.73 \times 10^{8} \mathrm{KgCO}_{2}$. When the percentage of EVs increases the total carbon emissions produced decreases; it follows a negative correlation. The smallest amount of carbon produced $\left(\mathrm{KgCO}_{2}\right)$ is when the percentage of EVs is $30 \%$, with a value of $1.49 \times 10^{8} \mathrm{KgCO}_{2}$. Furthermore the total carbon emissions falls $13.48 \%$ from when there is no EVs to when there is $30 \% \mathrm{EVs}$.

(ii) Figure 6 shows the result of the second scenario. The second scenario uses an average car carbon emission data from the year 2015 where the total carbon dioxide produced is $125 \mathrm{gCO}_{2}$ per car per $\mathrm{Km}$. The overall carbon emissions reduces by a considerable amount of approximately 54 million $\mathrm{kgCO}_{2}$ which is due to a more efficient engine that produces less $\mathrm{CO}_{2}$ per km travelled. However, the reduction of $\mathrm{CO}_{2}$ caused by an increasing number of EVs is smaller at only $6.67 \%$ compared to $13.48 \%$ using the data from 2000

(iii) Figure 7 shows the result of the third scenario. This scenario predicts that the carbon emissions produced by a vehicle from the 'normal' fleet will be less than in the previous scenarios. The level of carbon emissions predicted is $95 \mathrm{gCO}_{2} \mathrm{per}$ $\mathrm{Km}$. This improved efficiency produces the fewest carbon emissions $\left(\mathrm{Kg} \mathrm{CO}_{2}\right)$ of only $9 \times 10^{7}$ even when no electric cars are present in the fleet. However, there is an unexpected result in this case as the total carbon emissions produced $\left(\mathrm{KgCO}_{2}\right)$ does not decrease as the percentage of EVs increases from 5-30\%. The number of carbon emissions stays constant in this case; this suggests that the electricity carbon emission factor is restricting the carbon emissions from improving.

\section{Discussions}

Figure 8 shows the impacts that a large number of EVs could have on the grid if this uncontrolled charging took place. An increased number of EVs would only add to the pressure on the grid.

The results gained by increasing market penetration levels for all of the vehicles to be fully charged shows the daily electricity demand by the vehicles. When there are very high levels of market penetration the energy required by the cars reaches very high levels. Even though it is unrealistic to assume that all of the cars are recharge in full capacity in dailybasis, nevertheless it shows the potential energy these cars may require should uncontrolled charging take place. When all of the vehicles are recharged daily at $25 \%$ penetration, they require $40 \mathrm{GW}$ of electricity. This value would add a huge spike to the electricity demand and need to be accounted for.

For the electricity grid to cope with an increased number of EVs on the road the cars would need to be charged in a controllable environmental. Controlled charging would be a way of charging cars when the grid has a lower level of electricity demand and therefore alleviating pressure on the electric grid. Co-ordinating charging can be done by a smart metering system. If the system was not adapted by this system to charge at times when the demand is less the grid would need to be enforced. Upgrading the grid would be the only option to cope with increased loads and voltage drops by heavy charging. Both the scenarios will apply added costs to distribution system operators and eventually the customers.

From the results it is clear to see that the introduction of EVs reduces the overall carbon emissions mainly due to the fact that a proportion of petrol and diesel cars that are replaced by the EVs. There are European guidelines in place to ensure the reduction of carbon emissions from petrol and diesel car engines. Therefore the overall amount of carbon produced by petrol and diesel cars will decrease over time as engines become more efficient. As shown in Figure 7, in the year of 2020, where the normal car engines are predicted to have the most efficient engine, the overall carbon emissions were reduced. However, the carbon emissions only reduce up to a certain level and then the percentage reduction of emissions due to an increasing amount of EVs has less effect. 
The overall reason for the percentage reduction of $\mathrm{CO}_{2}$ from an increased number of EVs not increasing further; is down to the fuels used to produce the electricity that power the EVs. Figure 9 shows a provisional UK energy mix from 2014. This energy mix is made up from many fuel sources that produce large emissions of carbon dioxide, for example $29.1 \%$ of electricity is produced by coal. Currently in the UK electricity produced from renewable sources accounts for a small proportion of the total electricity produced; around 19.2\%. In 2014 the electricity produced by coal outweighs the electricity produced by renewable resources. The climate change act 2008 [45] set by the UK government aims to cut carbon emissions by $80 \%$ by 2050 . To achieve this aim the electricity produced by low-carbon renewable resources in the UK is going to increase and the electricity produced by high-carbon fossil fuels is going to decrease. As a result the $\mathrm{CO}_{2}$ emissions produced by electricity generation in the future will be less and therefore EVs will be less carbon emissive than petrol and diesel vehicles. Furthermore as the number of EVs increases and replaces many petrol and diesel cars, this means the sustainability of the transport sector will improve.

\section{Conclusion And Future Work}

This research has investigated the impact that a large increase of EVs would have on the current electricity grid. It has also evaluated the effect these vehicles will have on carbon emissions produced from passenger cars.

As uncertainty for the research was the future market penetration of EVs. There are many degrees of freedom when attempting to predict future penetration levels in the UK; results from previous papers are very different. Therefore, the project used a model which hypothesized different market penetration levels of EVs to predict the total electricity a fully electric fleet would require.

The results from the project show that even when there is a very high level of market penetration from EVs the overall effect on annual energy consumption may seem minimal. Conversely the effect that EVs may have on the electricity grid is actually dependent on the time of day they are plugged in. Results from the investigation show that when EVs occupy $25 \%-30 \%$ of the total car fleet they can have a substantial effect on the power demand. Therefore, it can be drawn that measures need to be put in place to control charging times of EVs; this as a result would help restrict the total daily power demand.

This work evaluates the potential carbon reductions by increasing the number of EVs in the UK. The results showed that the electricity grid would need to be upgraded as there were only positive results gained when the EVs occupied a large market share. As the UK's energy mix is predicted to improve drastically it can be concluded that EVs do offer a realistic alternative to internal combustion engine powered cars in terms of $\mathrm{CO}_{2}$ and electric grid available capacity.

This investigation aimed to evaluate the overall sustainability of EVs; overall it has been a successful indictor of the potential $\mathrm{CO}_{2}$ reductions and the demands on the electric grid. However it is essential to note that an extensive use of EVs may not actually contribute to the development of a sustainable transportation system. Even though EVs can reduce environmental stresses of road transportation this is only one aspect of sustainable development. In order to progress towards a new paradigm of sustainable development more effort is needed to pursue collective transport.

Future work to be undertaken needs to follow sustainable mobility. Firstly, there is limited peer-reviewed scientific literature specifically assessing techno-economics of a battery recharging infrastructure. Research needs to be work alongside industry and community stakeholders to support education and green vehicle readiness. Research needs to be actively involved in developing standards to support grid-friendly charging solutions to accommodate future growth of the vehicles.

\section{Declarations}

\section{Supplementary Information}

Page $11 / 19$ 
No supplementary information.

\section{Acknowledgements}

This work was not supported by any funding agency.

\section{Availability of data and materials}

I would like to declare that the work described was original research that has not been published previously, and not under consideration for publication elsewhere, in whole or in part.

\section{Author contribution}

WM Cheung is the sole author of this manuscript.

\section{Ethics approval and consent to participate}

The author has approved and participated the manuscript that is enclosed.

\section{Consent for publication}

Publication has been approved by the author.

\section{Competing interests}

The author declares no competing interests.

\section{References}

1. Adnan N, Nordin SM, Rahman I, Vasant P, Noor MA (2018) An overview of electric vehicle technology: a vision towards sustainable transportation. Intelligent transportation and planning: breakthroughs in research and practice, pp. 292-309

2. Constantine S; Meisterling (2008) Life Cycle Assessment of Greenhouse Gas Emissions from Plug-in Hybrid Vehicles: Implications for Policy. Environ Sci Technol 42:3170-3176

3. Doucette RT, McCulloch MD (2011) Modeling the CO2 emissions from battery electric vehicles given the power generation mixes of different countries. Energy Policy 39(2):803-811

4. European Commission; "Renewable Energy: Moving towards a low carbon economy” 2020; Renewable energy target. Available: https://ec.europa.eu/energy/en/topics/renewable-energy. Accessed in November 2020

5. Global EVO 2019., 2019. https://www.iea.org/reports/global-ev-outlook-2019. Accessed in November 2020

6. Harris A (2009) Charge of the electric car [electric vehicles]. Engineering Technology 4(10):52-53

7. Hadley SW, Tsvetkova AA (2009) Potential impacts of plug-in hybrid electric vehicles on regional power generation. The Electricity Journal 22(10):56-68

8. Hawkins TR, Gausen OM, Strømman AH (2012) Environmental impacts of hybrid and electric vehicles-a review. The International Journal of Life Cycle Assessment 17(8):pp.997-1014

9. Hromádko J, Miler P (2012) Environmental benefits analysis of electric vehicles in the Czech Republic. Transportation Research Part D: Transport Environment 17(3):251-255

10. International Electricity Factors., 2018. https://www.carbonfootprint.com/international_electricity_factors.html. Accessed in September 2020

11. Morgan JP, News A (2018) https://www.jpmorgan.com/global/research/electric-vehicles. Accessed in September 2020 12. Juul N, Meibom P (2011) Optimal configuration of an integrated power and transport system. Energy 36(5):3523-3530 
13. Kapustin NO, Grushevenko DA (2020) Long-term electric vehicles outlook and their potential impact on electric grid. Energy Policy 137:111103

14. Kiviluoma J, Meibom P (2011) Methodology for modelling plug-in electric vehicles in the power system and cost estimates for a system with either smart or dumb electric vehicles. Energy 36(3):1758-1767

15. Köne A, Büke T (2010) Forecasting of CO2 emissions from fuel combustion using trend analysis. Renew Sustain Energy Rev 14(9):2906-2915

16. Li J, Wang X (2012) Energy and climate policy in China's twelfth five-year plan: A paradigm shift. Energy Policy 41:519528

17. Liu F, Tait S, Schellart A, Mayfield M, Boxall J (2020) Reducing carbon emissions by integrating urban water systems and renewable energy sources at a community scale. Renewable and Sustainable Energy Reviews, 123, p.109767

18. Martinez CM, Hu X, Cao D, Velenis E, Gao B, Wellers M (2016) Energy management in plug-in hybrid electric vehicles: Recent progress and a connected vehicles perspective. IEEE Trans Veh Technol 66(6):4534-4549

19. McCarthy RW, Yang C, Ogden J (2007) Impacts of electric-drive vehicles on California's energy system. Institute of Transportation Studies, UC Davis, pp 1-19

20. Paladugula AL, Kholod N, Chaturvedi V, Ghosh PP, Pal S, Clarke L, Evans M, Kyle P, Koti PN, Parikh K, Qamar S (2018) A multi-model assessment of energy and emissions for India's transportation sector through 2050. Energy Policy 116:1018

21. Park C (2018) A Study of Smart Grid Effects on Electric Vehicle Management Considering the Change of the Power Capacity Mix. The Open Transportation Journal, 12(1)

22. Perujo A, Ciuffo B (2009) Potential impact of electric vehicles on the electric supply system. European Commission Joint Research Centre, Institute for Environment and Sustainability

23. Putrus GA, Suwanapingkarl P, Johnston D, Bentley EC, Narayana M, 2009, September. Impact of electric vehicles on power distribution networks. In 2009 IEEE Vehicle Power and Propulsion Conference (pp. 827-831). IEEE

24. Razeghi G, Brown T, Samuelsen GS (2011) The impact of plug-in vehicles on greenhouse gas and criteria pollutants emissions in an urban air shed using a spatially and temporally resolved dispatch model. J Power Sources 196(23):10387-10394

25. Renewables, Chapter 6 : "RENEWABLE SOURCES OF ENERGY” Available:

https://www.gov.uk/government/uploads/system/uploads/attachment_data/file/450298/DUKES_2015_Chapter_6.pdf. Accessed in August 2019

26. Richardson P, Flynn D, Keane A (2011) Optimal charging of electric vehicles in low-voltage distribution systems. IEEE Trans Power Syst 27(1):268-279

27. Richardson DB (2013) Electric vehicles and the electric grid: A review of modeling approaches, Impacts, and renewable energy integration. Renew Sustain Energy Rev 19:247-254

28. Shafiee S, Fotuhi-Firuzabad M, Rastegar M (2013) Investigating the impacts of plug-in hybrid electric vehicles on power distribution systems. IEEE Transactions on Smart Grid 4(3):1351-1360

29. Shao S, Pipattanasomporn M, Rahman S, 2009, July. Challenges of PHEV penetration to the residential distribution network. In 2009 IEEE Power \& Energy Society General Meeting (pp. 1-8). IEEE

30. Sithole H, Cockerill TT, Hughes KJ, Ingham DB, Ma L, Porter RTJ, Pourkashanian M (2016) Developing an optimal electricity generation mix for the UK 2050 future, 100. Energy, pp 363-373

31. Takács Á, Rudas I, Bösl D, Haidegger T (2018) Highly automated vehicles and self-driving cars [industry tutorial]. IEEE Robotics Automation Magazine 25(4):106-112

32. Thiel C, Perujo A, Mercier A (2010) Cost and CO2 aspects of future vehicle options in Europe under new energy policy scenarios. Energy policy 38(11):7142-7151 
33. Transport Statistics Great Britain (2018) https://www.gov.uk/government/statistics/transport-statistics-great-britain2020. Accessed March 2019

34. Wu X, Freese D, Cabrera A, Kitch WA (2015) Electric vehicles' energy consumption measurement and estimation. Transportation Research Part D: Transport Environment 34:52-67

\section{Figures}

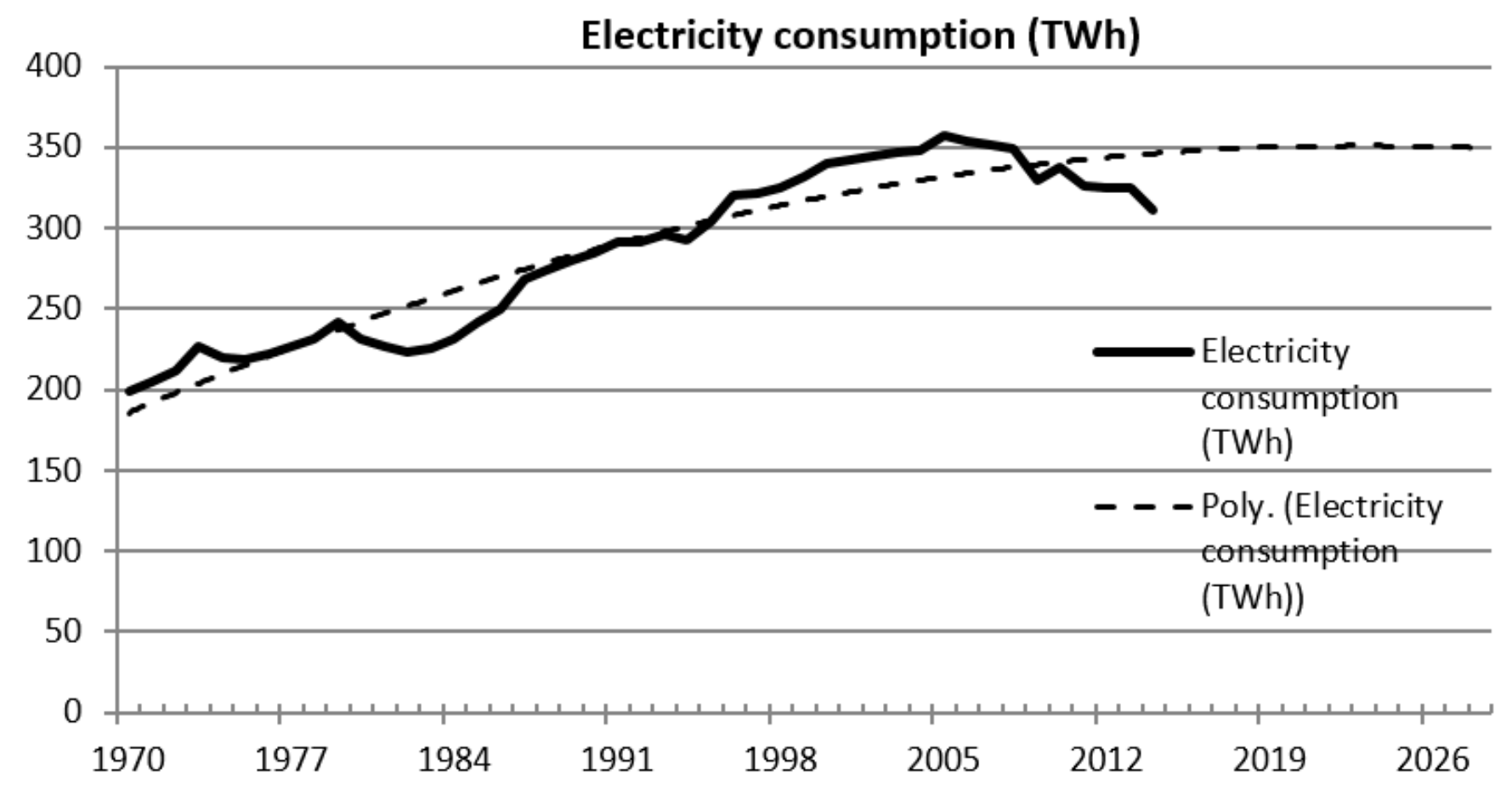

Figure 1

Actual and predicted energy consumption UK 1970-2030 
Total Number of cars in the UK (1000)

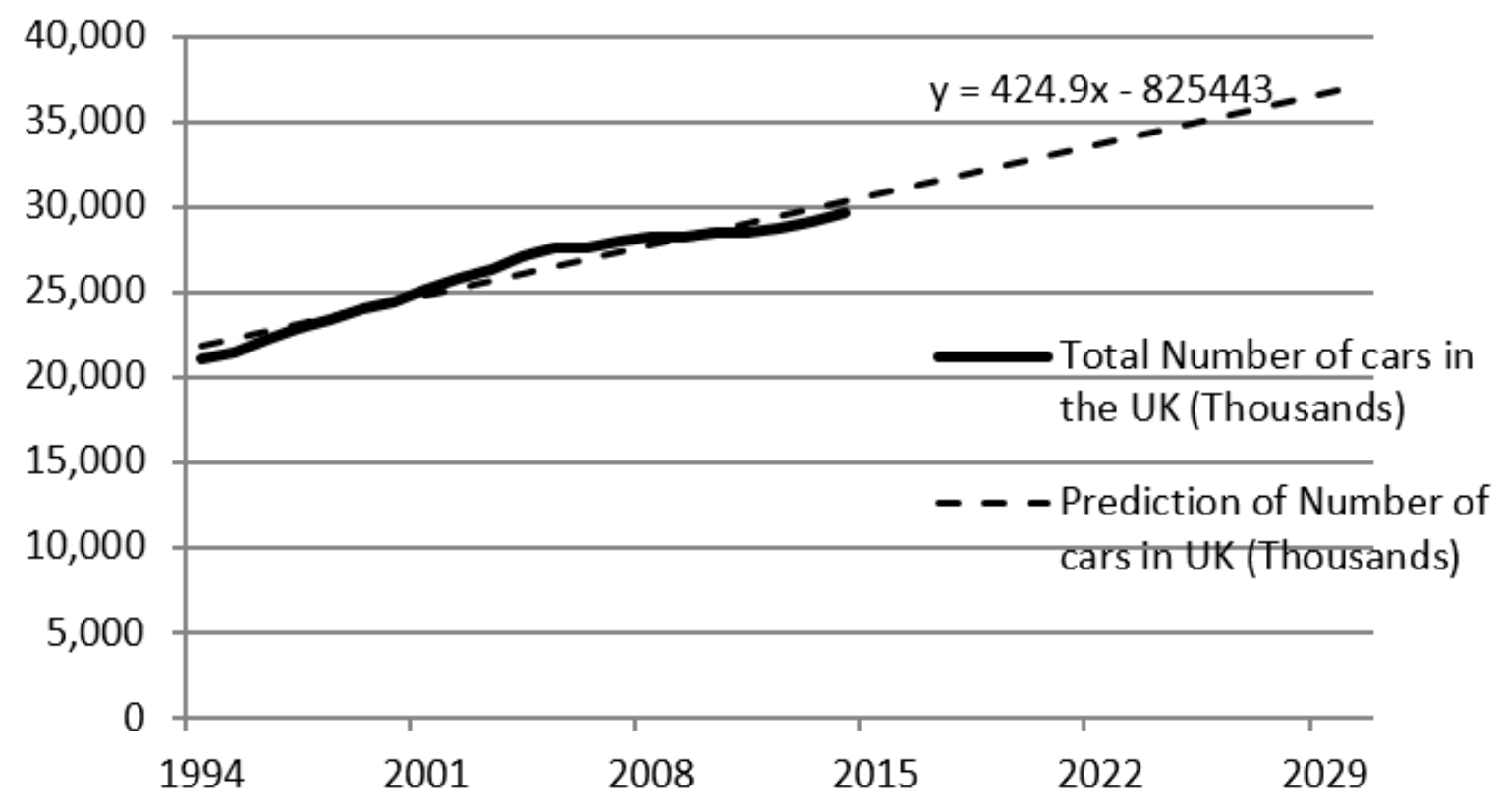

Figure 2

Total and predicted number of Cars in the UK

\section{Electricty Demand Prediction}

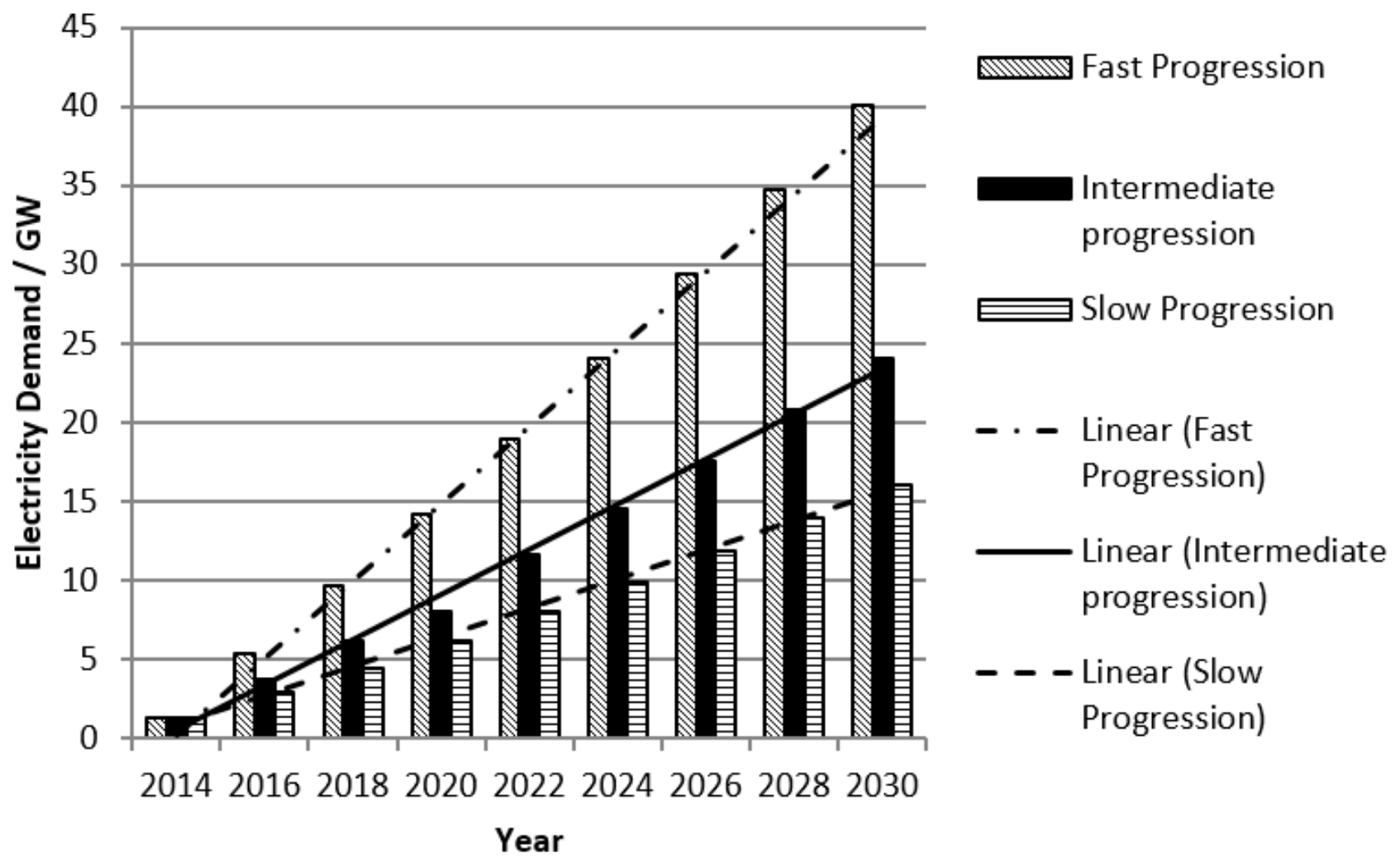

Figure 3 


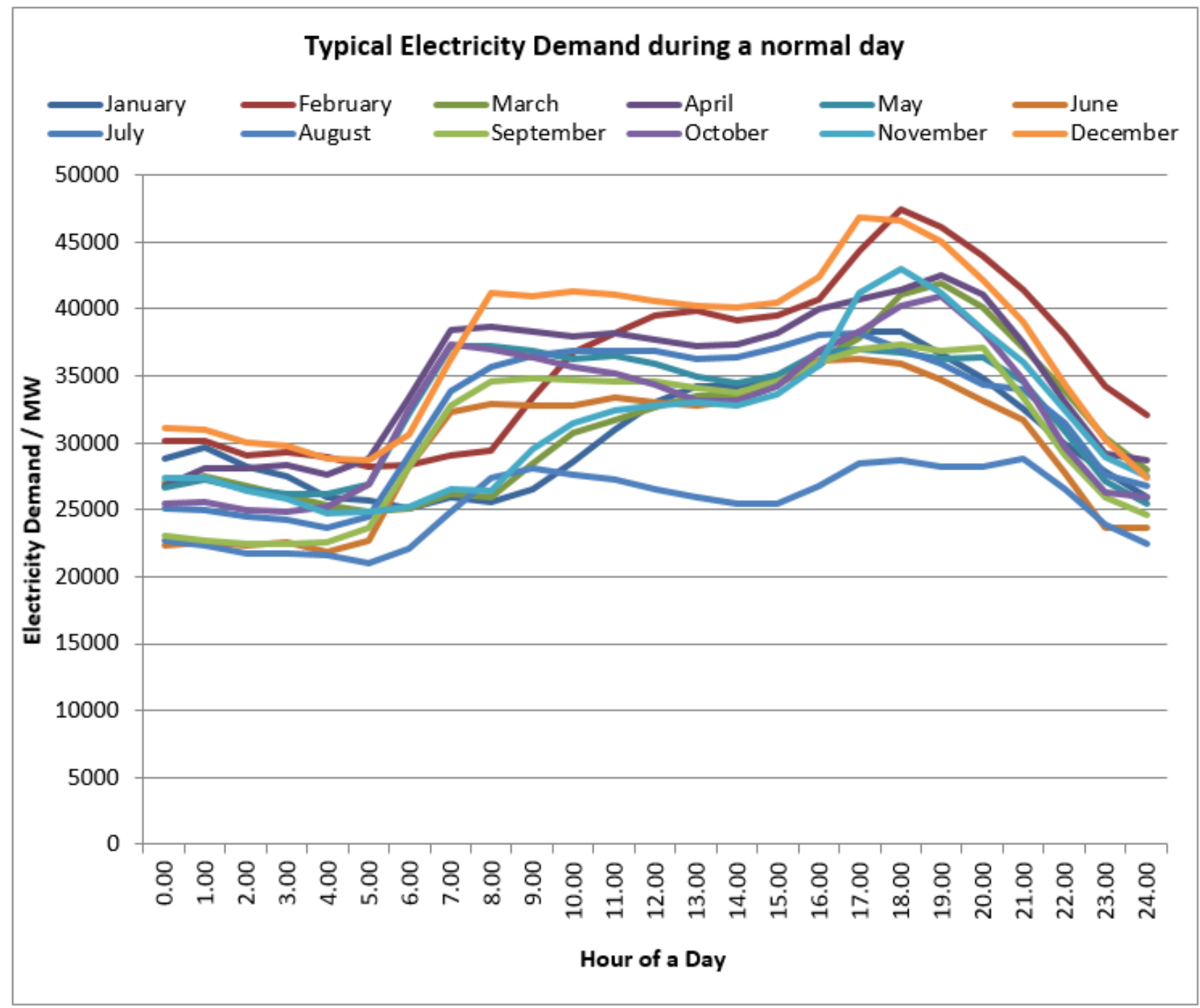

Figure 4

Daily Energy Demand UK (www.gridwatch.templar.co.uk) 
Carbon emmisions produced by car fleet with changing levels of electric vehicles

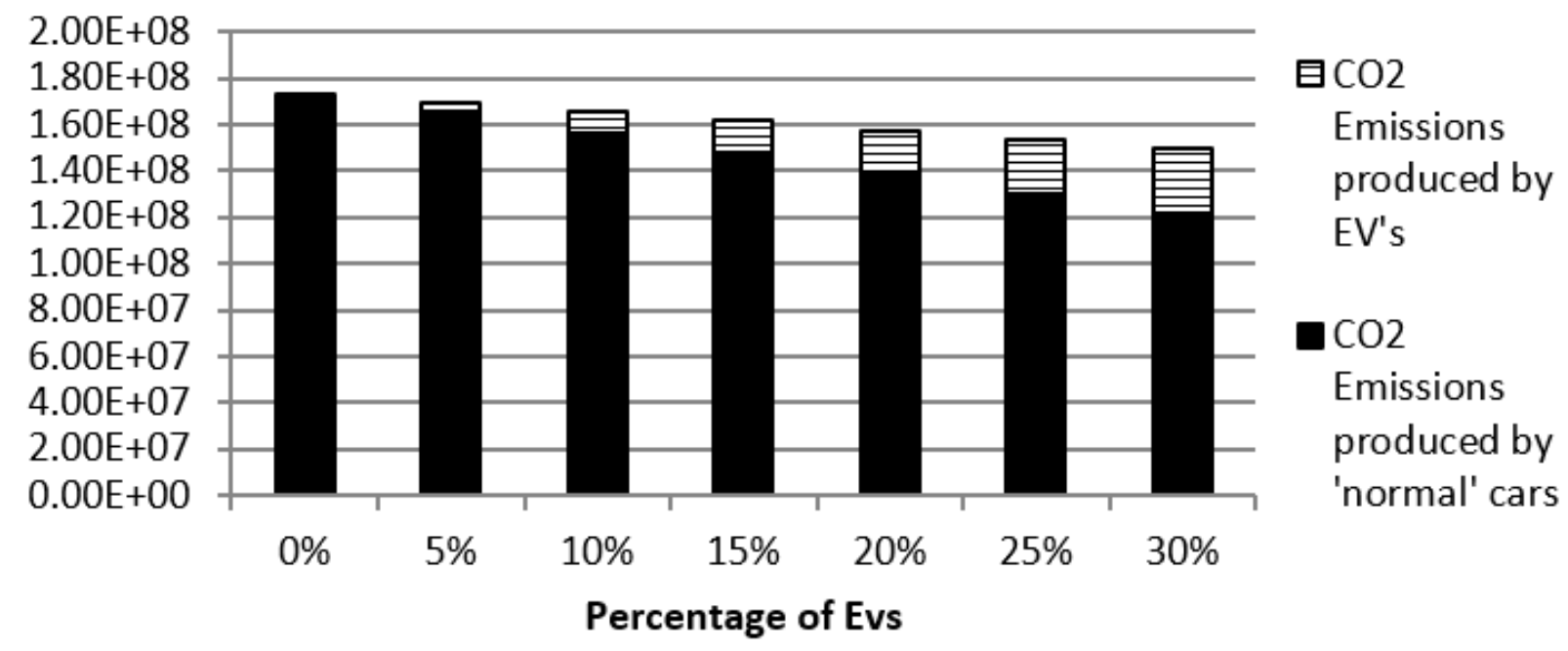

Figure 5

Carbon Emissions when normal cars $181 \mathrm{~g} \mathrm{CO} 2$ km

Carbon emissions produced by car fleet with changing levels of electric vehicles

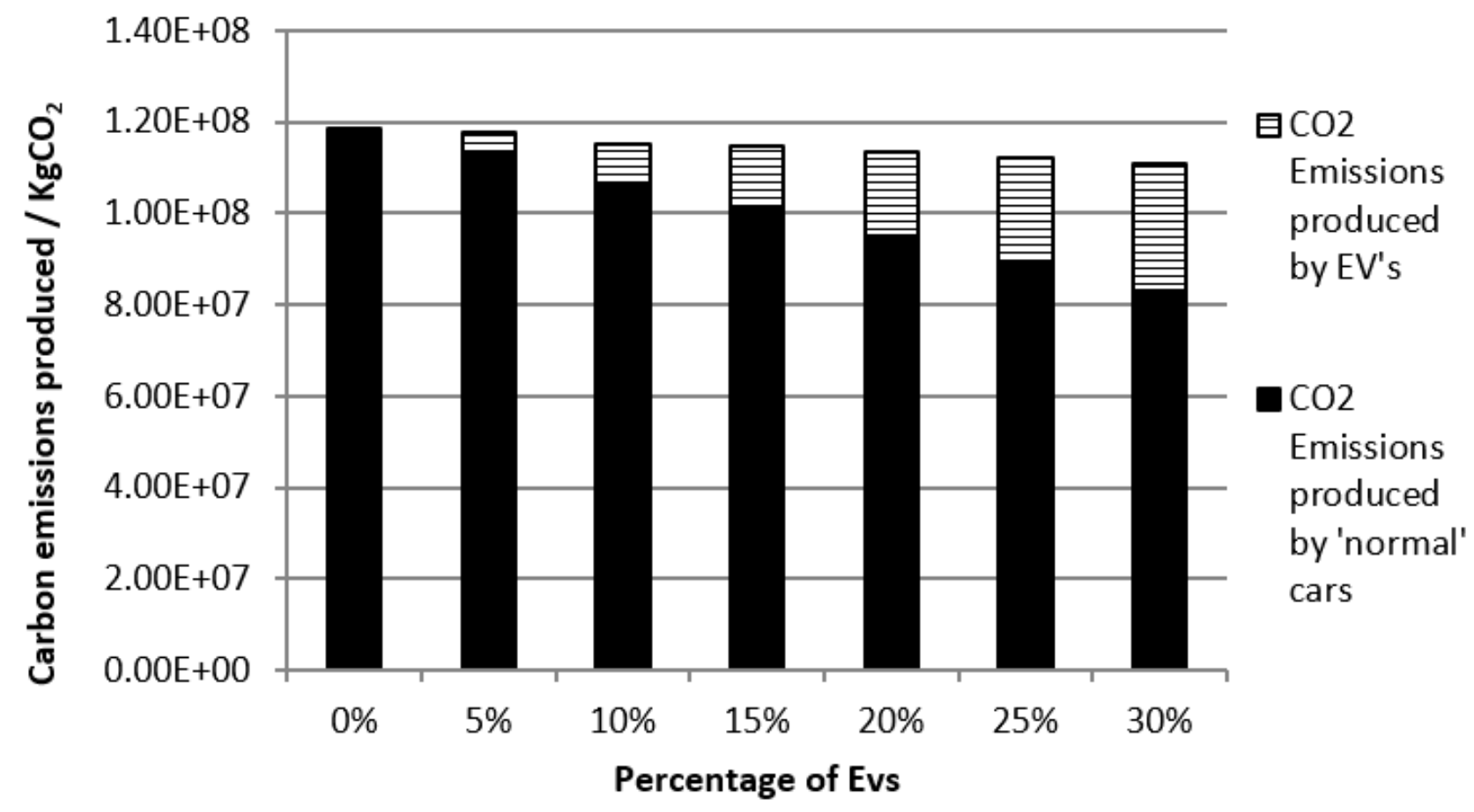

Figure 6 


\section{Carbon emissions produced by car fleet with changing levels of electric vehicles}

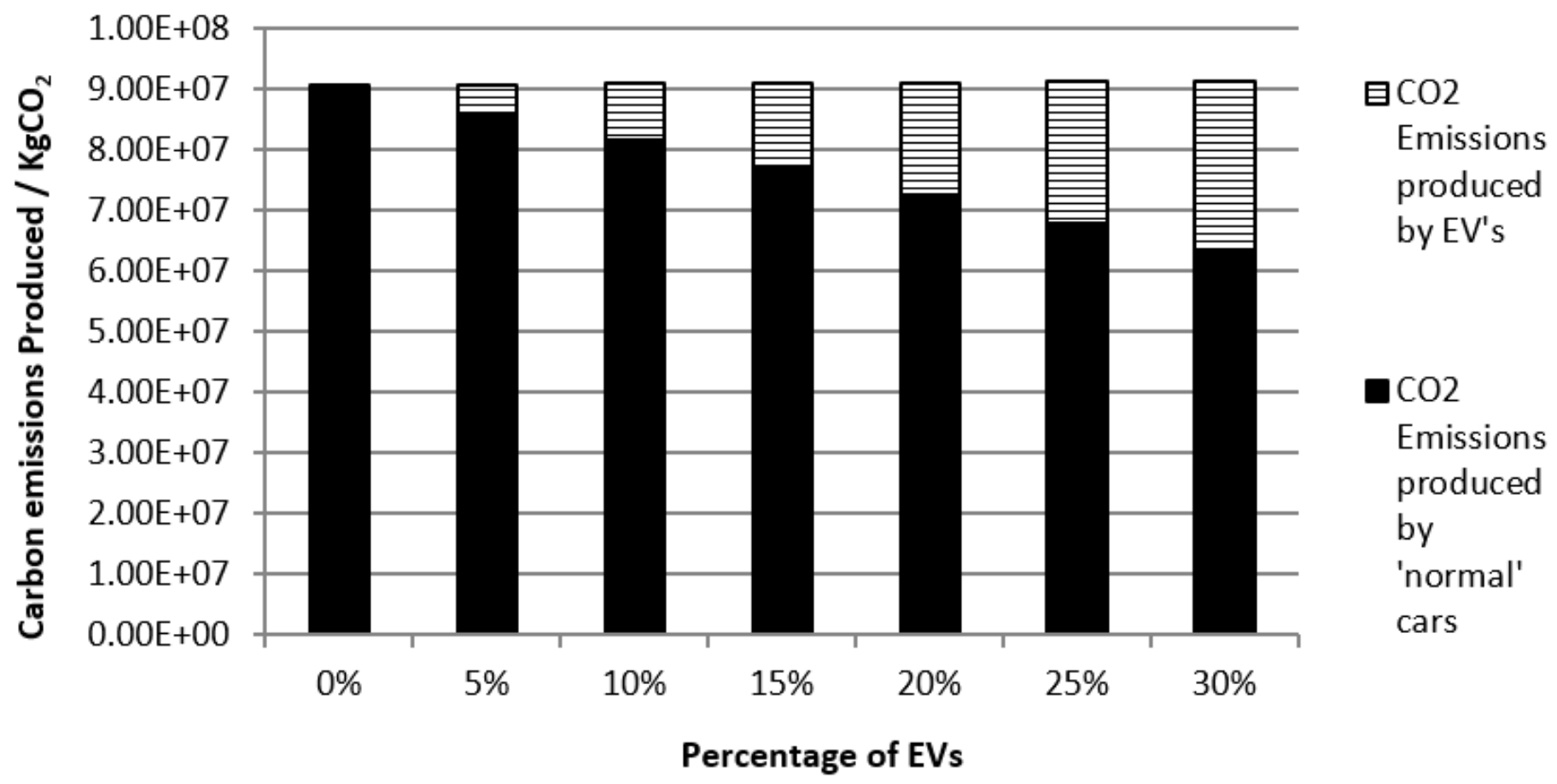

Figure 7

Carbon Emissions when normal cars 95g CO2 Km

Potential Un-controlled charging Demand

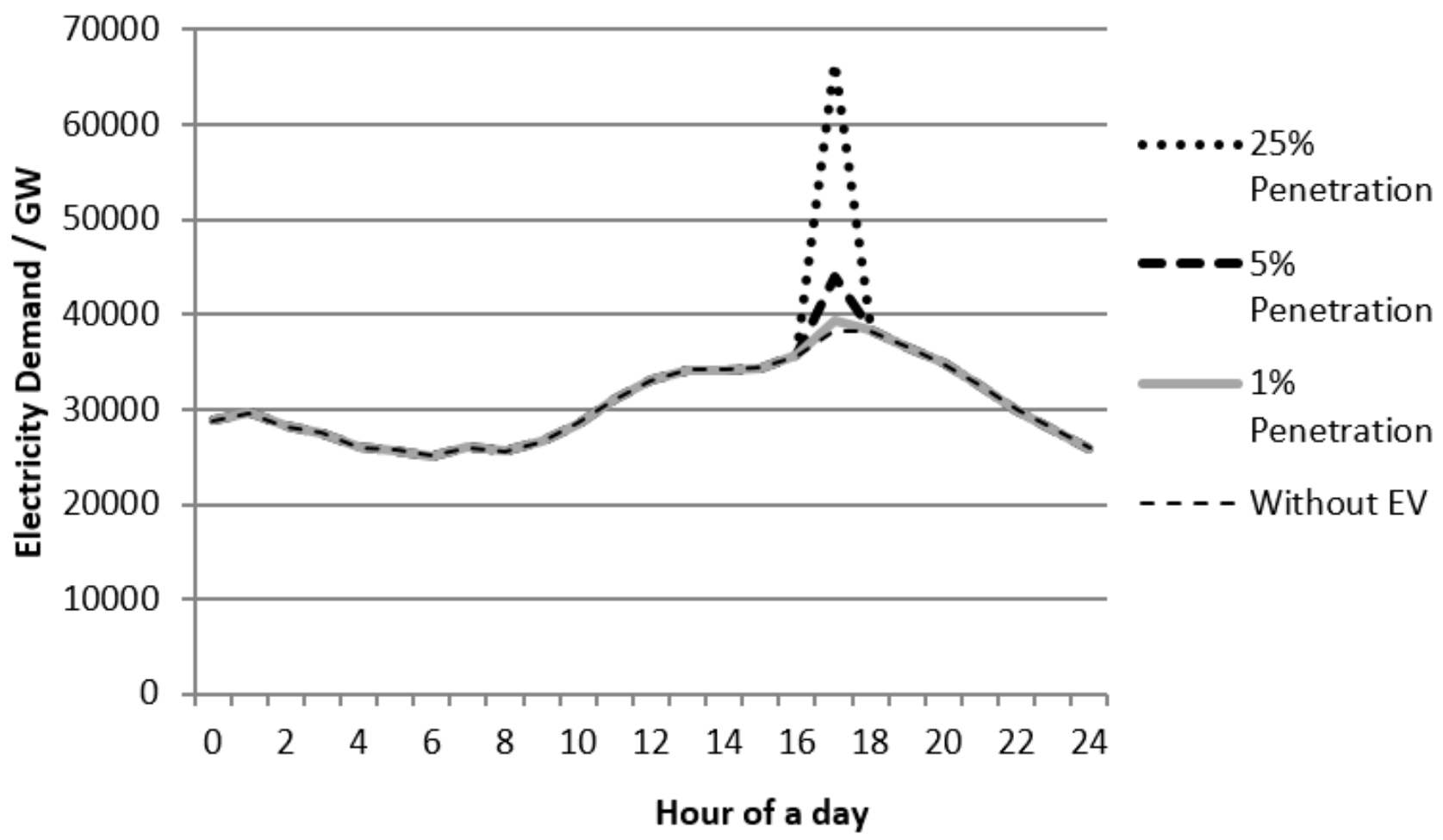

Figure 8 


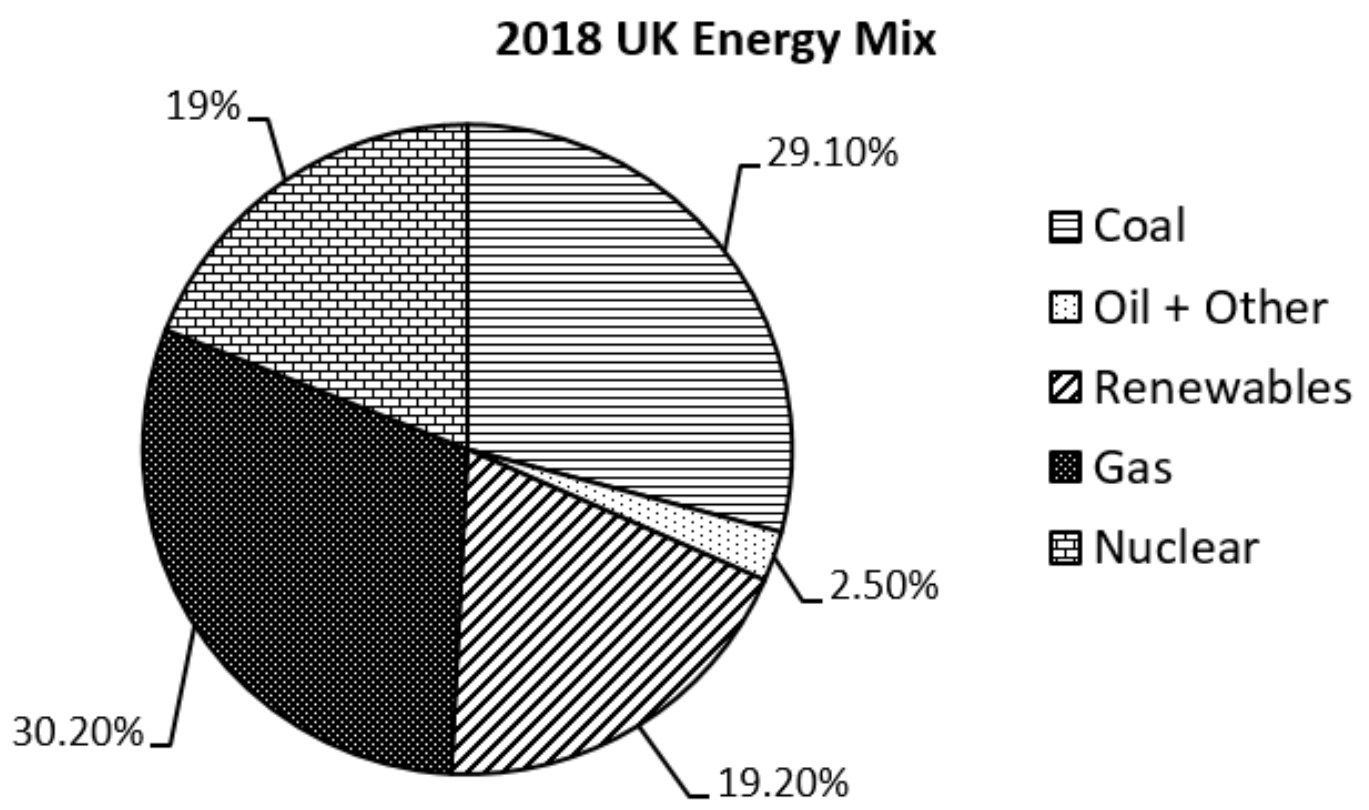

Figure 9

2018 UK Energy Mix 